\title{
Las espacialidades en la investigación clínica médica. El Instituto de Investigaciones Médicas de la Universidad de Buenos Aires, 1957-1976
}

The Role of Space in Clinical Medical Research. The Instituto de Investigaciones Médicas of the Buenos

Aires University, 1957-1976

As espacialidades na investigação clínica médica. 0 Instituto de Investigaç̃ões Médicas da Universidade de Buenos Aires, 1957-1976

Lucía Ana Romero PhD'

Recibido: marzo 3 de $2016 \bullet$ Aceptado: junio 19 de 2016

Doi: https://dx.doi.org/10.12804/revsalud14.03.2016.11

Para citar este artículo: Romero LA. Las espacialidades en la investigación clínica médica. El Instituto de Investigaciones Médicas de la Universidad de Buenos Aires, 1957 -

1976. Rev Cienc Salud. 2016;14(3):439-452 doi:

\section{Resumen}

Objetivo: este trabajo trata sobre la configuración de prácticas de transmisión, producción y uso de conocimiento científico en términos del establecimiento de diferentes espacialidades en la investigación clínica médica. Desarrollo: se reconstruyen las actividades desarrolladas en el Instituto de Investigaciones Médicas (IIM) de la Universidad de Buenos Aires, durante la dirección de Alfredo Lanari entre 1957 y 1976, por medio del uso de material documental y de archivo y del análisis de entrevistas realizadas a protagonistas de la época. Conclusiones: (i) la diferenciación y a la vez la interconexión de espacialidades fueron elementos centrales de la ideología y cultura institucional del Instituto de Investigaciones Médicas Lanari; (ii) los distintos espacios tales como la sección trasplantes y el servicio de diálisis hicieron del Instituto un lugar con reputación nacional e internacional al tiempo que otorgaron identidad y autoridad hacia su interior; (iii) la construcción de autoridad y jerarquía cognitiva implicó la puesta en juego de un conjunto de espacialidades tales como el ateneo y las residencias médicas y (iv) las formas de enseñanza y reclutamiento implementadas supusieron una nueva ecología común.

Palabras clave: investigación clínica médica, espacialidad, prácticas científicas.

1 conICET, Instituto de Estudios Sociales de la Ciencias y la Tecnología (IESCT) de la Universidad Nacional de Quilmes (Unq) y Facultad de Ciencias Sociales, Universidad de Buenos Aires. Correo electrónico: laromero@unq.edu.ar 


\begin{abstract}
Objective: this paper study how scientific knowledge is, transmitted, produced and used in terms of the establishment of different spaces, in clinical and medical research. Content: we analyze the way research activities developed at the Instituto de Investigaciones Médicas de la Universidad de Buenos Aires, during Alfredo Lanari was its director, between 1957 and 1976, using data from documental archives and form personal interviews. Conclusions: (i) differentiation and interconnection of spaces were central issues of the institutional culture and ideology of the Instituto de Investigaciones Médicas Lanari; (ii) different spaces such as transplant section and dialysis service help the Institute to build itself as a place with national and international reputation as well as to reinforce internal authority and identity; (iii) cognitive authority and hierarchies construction mobilized a set of spaces such as medical residencies and seminars (the ateneo) and (iv) teaching and recruitment reforms involved a new common ecology.
\end{abstract}

Keywords: Clinical medical research, space, scientific practices.

\title{
Resumo
}

Objetivo: este trabalho trata sobre a configuração de práticas de transmissão, produção e uso de conhecimento científico em termos do estabelecimento de diferentes espacialidades na investigação clínica médica. Desenvolvimento: reconstroem-se as atividades desenvolvidas no Instituto de Investigações médicas (IIM) da Universidade de Buenos Aires, durante a direção de Alfredo Lanari entre 1957 e 1976, através do uso de material documental e de arquivo e da análise de entrevistas realizadas a protagonistas da época. Conclusões: (i) a diferenciação e ao mesmo tempo a interconexão de espacialidades foram elementos centrais da ideologia e cultura institucional do Instituto de Investigações Médicas Lanari, (ii) os distintos espaços tais como a seção transplantes e o serviço de dialise fizeram do Instituto um lugar com reputação nacional e internacional ao tempo que outorgaram identidade e autoridade a seu interior, (iii) a construção de autoridade e hierarquia cognitiva implicou a posta em jogo de um conjunto de espacialidades tais como o ateneu e as residências médicas e (iv) as formas de ensino e recrutamento implementadas supuseram uma nova ecologia comum.

Palavras-chave: Investigação clínica médica, espacialidade, práticas científicas.

\section{Introducción}

En este trabajo se reflexiona sobre la configuración de prácticas de producción, transmisión y uso de conocimiento científico en términos del establecimiento de espacialidades en la investigación clínica médica, desarrollada en el Instituto de Investigaciones Médicas, durante la dirección de Alfredo Lanari entre 1957 y 1976. Este proceso se llevó adelante al configurar condiciones espaciales muy peculiares. Estas consistieron en la diferenciación y, a la vez, la interconexión del laboratorio experimental con la sala de internación, el consultorio hospitalario y el aula docente, teniendo como resultado una diversidad de prácticas: experimentales, de atención clínica y de docencia, claramente diferenciadas y a la vez estrechamente interdependientes entre sí. Esta dinámica espacial doble 
fue el corazón de la ideología institucional que mantuvo la dirección de Lanari en el IIM durante su gestión y su correspondiente materialización en ciertos roles y modalidades de trabajo médico. Este trabajo busca dar respuestas a las siguientes preguntas: ¿qué implicó construir, aplicar y trasmitir conocimiento en un lugar particular como el Instituto Lanari?, ¿qué tipo de realizaciones le dieron identidad y lo convirtieron en un legítimo espacio científico?

La investigación científica tiene sus singularidades, que imponen condiciones especiales en cuanto a sus arreglos en el espacio. Las prácticas científicas están atravesadas por instrumentos, equipos y aparatos de gran tamaño; así como también de animales, gases, sustancias, datos, personas, etc. La distribución y ocupación de este conjunto de elementos en el espacio físico, y entre la ubicación que ocupan los mismos investigadores, implican rutinas de trabajo, prácticas de cooperación, relaciones de autoridad-jerarquía. La relación entre la mesada y el escritorio en las ciencias experimentales, la circulación de un lugar al otro y la mayor ocupación y trabajo en uno u otro espacio revelan, entre otros elementos, las diferentes jerarquías y la división social del trabajo que se configura entre los practicantes y el orden del laboratorio. Pero a su vez, los diseños infraestructurales y espaciales son a la vez recursos de diferenciación y legitimación de la ciencia ante la sociedad. De hecho, el proceso de institucionalización de la ciencia moderna en gran medida refiere al modo por medio del que las prácticas científicas fueron asociadas con sitios específicos: el laboratorio universitario y más tarde el laboratorio hospitalario, y el público estatal.

El presente trabajo se estructura de la siguiente manera. Primero, se presenta una reflexión conceptual en torno a la cuestión espacial de la ciencia. Luego, la reflexión se focaliza en el análisis de la espacialidad como recurso ordenador del espacio de interacción cognitivo y social del Instituto, organizado sobre (i) las espacialidades del Instituto, (ii) su diseño cognitivo de interconexión permanente entre el laboratorio y la sala asistencial, (iii) los ateneos semanales como espacios de control interno y de construcción de reputación pública y (iv) los espacios de educación y reclutamiento de nuevos miembros, organizados alrededor de las residencias y las unidades docentes hospitalarias. Finalmente, las conclusiones intentan sistematizar las principales dinámicas puestas en juego en cada uno de estos planos.

\section{El lugar y la cuestión espacial en}

la ciencia:

La dimensión espacial de las actividades científicas ha sido tematizada por ciertas perspectivas dentro del campo de los Estudios Sociales de la Ciencia y la Tecnología. Es posible identificar cinco olas: en la primera, el positivismo y la filosofía racionalista de la ciencia entendían que analizar la ciencia significaba estudiar el carácter científico, abstracto y universal de la verdad científica, prestando nula atención al lugar específico donde esta se desarrollaba (1). En la segunda ola, al inicio de la década de 1970, las etnografías de laboratorio incursionaron en el laboratorio científico y mostraron cómo las contingencias específicas del lugar moldeaban el modo en que los científicos interpretaban los datos, usaban las máquinas, los instrumentos, llevaban adelante los experimentos, conformaban la validez científica, etc. Este giro en los estudios STS hacia el análisis de las prácticas acarreó consigo un interés por el contexto situado y local del trabajo científico, es decir, de los sitios de producción: los laboratorios, los museos, los centros de investigación $(2,3)$. Si bien, los etnógrafos redimensionaron el papel del carácter local de la construcción de conocimiento científico, el interés conceptual en el laboratorio como "un lugar" de la ciencia fue mínimo, es decir, el espacio, el lugar, no se 
constituyeron como un tema de interés por derecho propio $(1,3)$. La tercera ola refiere a un grupo de estudiosos del campo sTs que en la década de 1990 realizaron casos de estudio sobre una diversidad de sitios históricos, allí revelaron las diferentes precondiciones geográficas y materiales para producir conocimiento legítimo. Por ejemplo, cómo la importancia del proceso de testificación experimental pasó de los salones privados de los nobles a los laboratorios especializados en el siglo XIx. La cuarta ola es identificada con los teóricos del actor red en el sentido que su aporte conceptual apunta a mirar al lugar o al espacio de la ciencia en términos de una circulación. Así, el énfasis está colocado en la movilidad o en los flujos por donde se trasladan los actantes, lo que minimizó el significado geográfico de los lugares donde parten o llegan. El lugar aquí sería la circulación, la red, y no las posiciones fijas. La última y quinta ola se ubica temporalmente en los últimos años signados por la irrupción de las comunicaciones, internet y el aumento de movilidad de recursos humanos y de objetos de conocimientos. En este marco, de aparente irrelevancia de lo local, Henke y Gieryn intentan retomar los puntos señalados en la cuarta ola y, sin desmerecer la importancia de la circulación en términos de lugar y espacio de la ciencia, buscan volver a darle centralidad también al análisis de los puntos de partida y llegada de esos flujos de circulación, esto es, la localización geográfica y las materialidades situadas (1). En línea con este planteo, Salvatore coloca el énfasis en la cuestión del enraizamiento local de saberes, disciplinas y corrientes científicas de carácter trasnacional en los procesos de mundialización de la cultura (3).

Para el campo de la arquitectura y el urbanismo, la ciencia, como práctica y como institución, es un objeto sobre el cual se interviene sin contar muchas veces con una reflexión y abordaje sobre su especificidad. En la historia de la arquitectura y de los estudios urbanos existen algunas sistematizaciones históricas sobre los planes y estilos de construcción de los edificios de las universidades más tradicionales, así como también de centros e institutos científicos y laboratorios industriales, de investigación militar y universitaria (4-6). Amén de este tipo de recopilación, no abundan estudios empíricos que indaguen sobre la relación de esos diseños arquitectónicos, la espacialidad y el tipo de prácticas cognitivas y relaciones sociales que proliferaron en ellos, o del impacto en términos urbanos que tuvieron y tienen en sus zonas de influencia. Asimismo, ante la falta de desarrollo de una tradición de arquitectura científica y sí en cambio de una arquitectura hospitalaria y educativa, los laboratorios, institutos y edificios científicos en general han sido construidos bajo los criterios de aquellas; la ciencia desarrollada en general en sus inicios en la Argentina, y en parte del mundo, dentro de las universidades, ha sido teñida de su arquitectura escolar-educativa (7).

Retomando parte de estas discusiones, se entiende que el diseño arquitectónico, la infraestructura y la localización geográfica de un espacio donde se realiza ciencia tiene al menos un doble efecto; uno hacia afuera y otro hacia adentro. El primero remite a un proceso de legitimación pública. El conocimiento legítimo requiere de lugares legítimos. La autoridad de la ciencia y su fuerza cultural fue en ascenso al final del siglo xIx por medio de un proceso de diferenciación y especialización, basado en parte en las distinciones geográficas y arquitectónicas entre los lugares considerados apropiados para hacer ciencia y aquellos en los que no lo era. El bar, o el salón privado de un noble dejaron de ser lugares donde se desarrollaban tales prácticas (1).

El diseño arquitectónico, tanto de un edificio completo, de sus oficinas como de sus laboratorios, también tienen efectos hacia su 
vida interior, es decir, hacia el desarrollo de su cultura institucional y sus prácticas. Son factores que ayudan a moldear las prácticas que en ellos se desarrollan, en particular, el tipo de investigación que se realiza (experimental o no; disciplinar o interdisciplinaria; asociada con demandas y problemas del medio social o a problemas académicos), el modo por medio del que se entrenan y reclutan nuevos miembros, cómo socializan entre sí y con el resto del personal científico, las formas de vinculación y cooperación con centros y grupos extranjeros y el modo en que se construye identidad, autoridad y jerarquías científicas (grupal, institucional, individual) (8).

Sumado a esta noción de espacio, en tanto infraestructura y materialidad física, se entiende que este es también una forma de agencia. Las relaciones sociales son intrínsecamente espaciales y el espacio es un instrumento y una dimensión de la sociabilidad de los sujetos. En este sentido, es lo que los sujetos hacen y no meramente dónde están (9). Una idea afín a esta propuesta, se encuentra en Giddens en su concepto de "sede". En diálogo con los geógrafos, retoma alguno de sus aportes a propósito de la discusión sobre "el espacio, el territorio, el lugar, el contexto" donde suceden los fenómenos sociales, y opta por la noción de sede. Esta "denota el uso del espacio para proveer los escenarios de interacción y a su vez los escenarios de interacción son esenciales para especificar su contextualidad" (10, p. 151). Sedes son así las propiedades físicas y materiales (el lugar) y los usos (funciones) que a este se le dan y lo constituyen también en una sede.

\section{Las espacialidades dentro del Instituto}

En el marco de los proceso de renovación y modernización académica dados en la Universidad de Buenos Aires a partir de mediados de 1950, el 31 de julio de 1957 se creó el Instituto de Investigaciones Médicas, designándose a
Alfredo Lanari como su director full time ${ }^{2}$ (11). Esta creación implicó la institucionalización de un lugar de desarrollo renovado para la investigación clínica médica en la Argentina, de cara al campo de la investigación clínica local e internacional y de cara al campo científico y académico local en general. Pero al mismo tiempo, compartió rasgos con otras creaciones institucionales contemporáneas tales como el Instituto de Investigaciones Hematológicas de la Academia Nacional de Medicina o el Departamento de Física de la Facultad de Ciencias Exactas y Naturales FCEN de la UBA tales como la presencia, en todas estas construcciones institucionales, de un fuerte liderazgo científico, una ideología de trabajo de tiempo completo, una construcción programática de su organización cognitiva y el despliegue de fuertes estrategias para asegurar su reproducción ampliada (12, 13). Esto lo colocó en un clima de época local de renovación y de cambios en la disciplina médica de la segunda posguerra (entre los más importantes se encuentran el crecimiento del financiamiento en la investigación biomédica, los cambios en organización cognitiva y en la enseñanza médica tendientes a volverla más próxima a las ciencias experimentales) $(14,15)$.

El Instituto de Investigaciones Médicas se ubicó en el Centro de Investigaciones Tisiológicas y Tercera Cátedra de Semiología en el Hospital Tornú, existente desde 1937, y se organizó sobre la base del personal y de las líneas de investigación desarrolladas en la Tercera Cátedra de Clínica Médica de la Facultad de Medicina de la Universidad de Buenos Aires (16).

\footnotetext{
2 La historia de la investigación clínica en la Argentina y de la tradición de investigación que se conformó en el ım a partir de la figura de Alfredo Lanari constituyen los temas de mi tesis doctorado (Romero, 2011). Este artículo se nutre de una parte del trabajo empírico realizado en ella y nunca publicado.
} 
La organización asistencial se concibió como un insumo para las tareas de investigación, al tiempo que estas tuvieron siempre su inspiración y justificación en los problemas de salud recogidos de la práctica clínica.

El espacio físico del Instituto representó esta imbricación y diferenciación simultánea: el Instituto estaba compuesto por dos edificios; por un lado, estaba el anexo asistencial con los consultorios clínicos y las salas de internación y, por otro lado, se encontraba el ala de laboratorios. Ambos estaban separados por un estacionamiento y estaban unidos por el andar constante del personal que "cruzaba de un lado al otro" en forma permanente. Este accionar constituía un mandato institucional y un estilo de trabajo que impartía la dirección de Lanari (17).

Lo que hizo Lanari fue descentralizar los laboratorios, que en un hospital corriente están todos centralizados. Los puso a cargo de médicos que tenían que hacer investigación y ver pacientes. Creó una cosa única que consistió en que una persona que pipetea y mira aparatos y se configura cómo van a hacer cosas del laboratorio para la sala y también para su proyecto de investigación. Pero al mismo tiempo tenían la obligación de permanecer durante algunos meses en la sala viendo pacientes aunque no correspondieran a su especialidad, ese fue un factor muy importante porque mantenía a todos los especialistas con la cabeza bien abierta. Eso fue muy clave (18, p. 14).

La investigación clínica en el Instituto estaba, de este modo, gráfica y espacialmente representada en el mapa edilicio. La regla era que todo investigador y médico clínico cruzaran de un lado al otro en forma habitual, trazando puentes y vasos comunicantes entre la asistencia clínica y la investigación.
Otro lugar de encuentro entre las dos culturas fue el bar del Instituto, así como los ateneos semanales. Estos espacios de intercambio eran mayormente de tipo informal (los pasillos de los dos edificios, el estacionamiento, el bar), salvo los ateneos que tenían una regularidad semanal e implicaban una organización previa, con un expositor y una presentación a cargo para ser comentada por colegas (sobre ellos se hablará en las próximas secciones).

Si bien, la primera idea de Lanari respecto a la organización cognitiva del IIM consistió en concentrar sus capacidades de investigación en un tema o línea principal, ello no fue posible ya que se encontró con dos situaciones: (i) con médicos con inclinaciones por la investigación clínica de tiempo completo, que no encontraban otro espacio de radicación institucional en el país y (ii) con médicos que habiéndose entrenado en el extranjero (en el manejo de técnicas específicas de una especialidad) no contaban con espacios donde radicar su puesta en práctica al regresar al país. En este marco, abrió cualquier especialidad que estuviera en manos de médicos en alguna de estas dos situaciones. Así, a lo largo de los primeros cinco años, en el Instituto se inauguraron diecinueve secciones: Anatomía Patológica, Bacteriología, Cardiología, Cirugía Experimental, Clínica Médica, Endocrinología, Gastroenterología, Hematología, Hemoterapia, Hipertensión Arterial, Inmunología, Metabolismo, Fisiopatología Renal (más tarde Nefrología), Neumonología, Neurología, Radiología, Radioisótopos, Sustancias Vasoactivas y Urología (19). En un inicio, las diferentes secciones se organizaron bajo la forma de Departamentos y no todas incorporaron laboratorios de investigación; Hemoterapia, Radioisótopos, Urología, Gastroenterología, Clínica Médica, Cirugía y Cardiología se estructuraron desde un comienzo como servicios asistenciales y de diagnóstico que desarrollaban principalmente tareas de atención y exámenes de rutina a los enfermos de sala (20). En algunas ocasiones, operaban como 
servicios de apoyo a otras secciones que en cambio sí desempeñaban investigación, brindándoles a estas material de pacientes con los cuales trabajar. En este sentido, entre las más dinámicas se destacó Anatomía Patológica, con su acumulación de material de autopsias.

La diferenciación espacial de los investigadores según su especialidad derivó en la conformación de secciones, siguiendo una lógica burocrática y funcional que demandaba la organización social y cognitiva del Instituto. Las prácticas de conocimiento de cada especialidad (con sus instrumentos, equipos, aparatos, mesadas característicos) deslindaron una espacialidad propia que, al tiempo que se diferenciaba creando un orden administrativo, cognitivo y social (identificaciones de grupo), se interconectaba muy estrechamente en forma colaborativa con otras especialidades y en muchos casos con la práctica clínica asistencial. En particular, esto se verificó en el tema del trasplante renal, como se verá a continuación.

En suma, el Instituto conformó una sede, esto es, una estación para un amplio conglomerado de interacciones en el curso de su actividad cotidiana; el Instituto de investigación asociado con un hospital, como en este caso, se presentaba regionalizado en "anexos o alas", pisos, salas, habitaciones, departamentos/secciones, laboratorios, aulas, pasillos, el bar, el estacionamiento (10). Ello quiere decir que estaban zonificados de manera diferente en el tiempo y en espacio. Las aulas se utilizaban en ciertas horas del día para impartir clases a aprendices $\mathrm{y}$ en otros momentos para realizar ateneos $\mathrm{y}$ seminarios entre miembros del Instituto. A su vez, en ambos casos conformaban espacios de uso colectivo, mientras los laboratorios implicaban usos individuales y colectivos de trabajo de los investigadores exclusivamente. Las salas clínicas y las habitaciones, durante el día, eran espacios de interacción entre los profesionales (médicos, residentes, investigadores, enfermeras) y los pacientes, mientras que por la noche las habitaciones se configuraban en espacios donde los pacientes descansaban acompañados por residentes, enfermeras y médicos que rotaban según turnos ordenadamente pautados. Los pasillos, el estacionamiento y el bar durante el día se configuraban como sitios de encuentro y socialización profesional informales, aunque de suma significación en términos de la cultura de interconexión entre clínicos e investigadores, estimulada por la conducción de la institución.

\section{El diseño cognitivo: la interconexión entre el laboratorio y la sala} asistencial

Los trasplantes renales: El Instituto se constituyó como un lugar de derivación. Esto significaba que la población de enfermos internados no accedía por propia iniciativa sino que eran transferidos desde otros hospitales para la realización de estudios complejos, siendo cuidadosamente seleccionados, especialmente, los que portaban enfermedades difíciles o raras, en particular en lo atinente a patologías renales o inmunológicas. De este modo, sumado a la misión institucional comentada y al diseño y uso edilicio asentados en la interconexión entre investigación y clínica, en este aspecto también la organización asistencial se encontraba estrechamente ligada a la función de investigación, dado que los pacientes que se tomaban eran aquellos que podían ofrecer material humano de estudio para las líneas de investigación que se desarrollaban en el Instituto.

Asimismo, desde el punto de vista de la organización asistencial, el Instituto implementó, desde el comienzo, una modalidad en la que sus camas de internación no estaban diferenciadas por especialidades médicas. Los especialistas actuaban a través de los jefes de sala, lo cual proveía la unidad de criterio en la 
atención de los pacientes. "El especialista es un clínico general que sabe algo más en determinada rama de la medicina interna" (21, p.28). El recorrido y el trabajo en la sala de internación era una tarea obligatoria para todos los miembros, fueran clínicos o investigadores:

Lanari vino y me dijo: "Tiene que ir a la sala". "¿Cómo? ¿A la sala?" "Sí, tiene que ir a la sala". "Pero yo no sé Clínica Médica, doctor". "Tiene que ir a la sala". Bueno; fui a la sala. Tenía como residente a Jorge $H$, que ahora es un excelente químico y él era residente de 3er año. $Y$ entonces, cuando había que discutir algún enfermo yo le preguntaba: "H, decime cómo es esto". Y H me ayudaba un poco; me puse a estudiar Clínica Médica y estuve 3 años yendo, 6 meses por año, a la sala; estuve sometido a todo lo que sea problema clínico (22, p. 5).

De este modo, el espacio natural del investigador, el laboratorio, se ampliaba al de sala de los clínicos médicos y vicerversa. El mandato y la cultura institucional dictaban que los investigadores y clínicos debían transitar ambos sitios, mezclando sus intereses, saberes y prácticas (19).

Entre las especialidades que desarrollaron actividades de investigación y asistencia bajo dinámicas de alta interdependencia entre la investigación experimental (el laboratorio) y la clínica asistencial (la sala), la Sección de Trasplantes y el Servicio de Diálisis se destacaron dentro del conjunto. Esta imbricación data desde el inicio mismo de estas prácticas en el medio local en el contexto del cruce de dos tradiciones de investigadores diferentes.

Por cierto, las prácticas de trasplantes fueron posibles gracias a los contactos que el grupo del Instituto estableció con el grupo de John Putman Merrill en el Hospital Peter Bent Brigham en Boston (grupo pionero en el tema), por medio de las estadías de trabajo que realizaron miembros del Instituto y del aprendizaje y apropiación de técnicas y aparatos, como fue el primer riñón artificial —el Kolff Brighamtraído al país. Los primeros ensayos de trasplantes renales realizados en el Instituto datan de unos tres años después de los desarrollados por los grupos pioneros en 1954 (23).

También, los trasplantes fueron posibles debido a la acumulación que en ese campo de conocimiento existía previamente en el medio local: por un lado, aquella inserta en la tradición clínica de Vaccarezza en cuya Cátedra se habían investigado y realizado experiencias en dicho campo de estudio (injertos de aorta y pulmón), tanto por parte de Lanari como por otros miembros del Instituto que provenían de dicho espacio. Por otro lado, la tradición experimental, desarrollada por Houssay, y de ella especialmente las investigaciones realizadas entre 1930 y 1940 sobre fisiología del riñón (24).

Junto con Trasplantes, hubo dos secciones/especialidades que tuvieron un despegue de magnitud diferencial respecto al resto, en cuanto a trayectorias de su personal, temas investigados, su vinculación con problemas de salud públicos de envergadura social y, fundamentalmente, por haber aportado técnicas y conocimientos de mucha relevancia en la investigación y el tratamiento en trasplantes renales y diálisis. Estas fueron hematología e inmunología (24). A partir de mediados de la década de 1960, con la aparición de los desarrollos de los estudios de histocompatibilidad y con la aplicación de drogas inmunosupresoras, los procedimientos fueron tomando mayor complejidad y ampliando a su vez las posibilidades de realización $(25,26)$. En este marco, la Subsección Trasplantes comenzó a estrechar vínculos de trabajo con dos secciones afines y subsidiarias en lo atinente a analizar y dar 
respuestas a los problemas más usuales en trasplantes: aceptación y rechazo inmunogenético del órgano. Estas secciones fueron Hematología, Inmunología y Anatomía y Patología (27).

Asimismo, la Sección Diálisis creció a la par de la Subsección Trasplantes de la Sección Nefrología, ya que conformaba un servicio autónomo (asistía en diálisis a pacientes renales crónicos), pero también subsidiario de aquél, pues los pacientes que iban a recibir un trasplante eran dializados previamente, ya que ello generaba mejores condiciones para una intervención exitosa. Asimismo, eran dializados después de recibir el trasplante. Así, si bien existía una clara diferencia entre la sala de diálisis, de trasplantes y los laboratorios de fisiopatología renal, en términos de lo que se hacía en cada lugar como desde el punto de vista del instrumental y la aparatología existente, habían conexiones en forma de interdependencia funcional.

La diálisis, por ejemplo, implicaba procedimientos muy cuidadosos, casi artesanales, y una distribución espacial definida por el gran tamaño del aparato de diálisis (el caso de los primeros modelos como el Kolff), los profesionales y el paciente. El manejo del aparato Kolff requería de un equipo entrenado y bien coordinado de médicos, laboratoristas y enfermeras. Todos ellos debían conocer y monitorear no solo el funcionamiento mecánico (y sus potenciales problemas), sino todos los factores que intervenían en la diálisis. Los diferentes tubos que formaban el circuito cerrado que se colocaba entre arteria y vena debían ser armados cada vez, cuidando que no hubiera soluciones de continuidad por las que pudiera escaparse la sangre, entrar agua o aire al baño. Este preparado y procedimiento artesanal requería un monitoreo constante. La principal labor de los enfermeros era controlar la presión arterial, el pulso y la respiración del enfermo, los cuales debían medirse cada cinco minutos al comienzo de la diálisis y cada diez hacia el final. El armado del aparato demandaba más de dos horas y las diálisis duraban entre cuatro y seis (28).

En suma, los trasplantes renales y la diálisis implicaron específicas formas de espacialidad en su trabajo, fueron los temas de investigación y tratamiento que dinamizaron las mayores interrelaciones entre las especialidades médicas del Instituto, lo que generó una fuerte identidad de trabajo y autoridad cognitiva alrededor de esto y contribuyeron a que el Instituto se legitimara social y cognitivamente en relación con los enfermos renales y la comunidad científica local e internacional. A partir de 1960 el IIM se fue posicionando como el centro de referencia en investigación y tratamiento en diálisis y trasplantes renales también, por medio de la formación de profesionales y el establecimiento de relaciones con otras instituciones locales, en las que se fueron diseminando aquellos conocimientos y expertise -más tarde difundidos asimismo al resto del tejido hospitalario de la ciudad de Buenos Aires (29)—.

El ateneo semanal: un espacio de control interno y construcción de autoridad: La dinámica de trabajo entre los investigadores formados y en formación se basaba en la realización de ateneos semanales. Estos constituían un espacio de reunión donde los jefes de secciones y sus becarios discutían los casos clínicos y su vinculación con las investigaciones en curso. La rutina consistía en la realización de tres ateneos por semana: uno quirúrgico, donde se discutían los casos que iban a ser sometidos a una cirugía; otro clínico, donde se discutían los casos de pacientes con problemas de difícil diagnóstico $\mathrm{y} / \mathrm{o}$ tratamiento $\mathrm{y}$, por último; un ateneo anatomopatológico, donde se discutían los resultados de las autopsias con presencia de los clínicos que habían atendido al enfermo $(17,19)$. 
Los ateneos constituían, principalmente, un espacio de intercambio y aprendizaje no solo entre los investigadores formados sino también para aquellos estudiantes en formación, quienes tenían la obligación de asistir y participar. Esta función pedagógica de los ateneos residía centralmente en exponer los modos de intercambio, discusión y crítica, basados en la argumentación y la participación activa, así como también centrar el aprendizaje sobre los errores de la práctica clínica.

Sumado a esta función pedagógica, la dinámica semanal de los ateneos conformaba un espacio en el cual los jefes de secciones y el director del Instituto podían realizar un control y un seguimiento de la evolución de los casos clínicos complejos y del estado de actualización de los trabajos de investigación llevados adelante en las distintas secciones.

La dinámica y espíritu de los ateneos semanales, donde se reunían todos los especialistas a discutir un caso clínico, ponía en práctica también la visión y el desempeño que Lanari estimulaba dentro del Instituto, esto es, la prioridad de la figura del clínico sobre la del especialista:

Todas las semanas tenías que preparar un caso, presentarlo, discutirlo pero, a su vez, lo discutían todos los especialistas. Y ahí había gente muy buena [...] nos fuimos enseñando unos a los otros. Y estábamos ahí todo el día. Entonces, yo, si a las 4 de la tarde tenía alguna pregunta sobre Hematología, lo tenía a Sánchez Ávalos ahí. Fuimos todos formándonos y eso fue una tarea y fue el resultado de la decisión de Lanari de que todo el mundo tiene que saber Clínica Médica antes de ser un especialista (30, p.8).

Dos rasgos más caracterizaban a los ateneos: por un lado, la pretensión, promovida por Lanari, de la mayor intervención posible de médicos y residentes pero siempre a partir de razonamientos, diagnósticos y tratamientos fundados en la medicina experimental o anatomopatológica; por otro lado, la primacía de una clara jerarquía de autoridad y responsabilidad en cuanto a la toma final de decisiones en cada caso tratado: la del director del Instituto y, luego, la de los respectivos jefes de sección. Este escalonamiento de autoridad y responsabilidad era trasladada a otras relaciones entre jefes y subalternos en ámbitos diferentes al del espacio de los ateneos. Por ejemplo, en la sala de internación, en los laboratorios y en los espacios de docencia con la implementación del sistema de residencias y de la unidad docente hospitalaria (UDH), como se verá a continuación. La construcción de autoridad cognitiva y social y la conformación de relaciones de jerarquía también implicaban de este modo una forma de espacialidad a la vez que se configuraban en determinados espacios de interacción. En los ateneos semanales la autoridad entre jefes y aprendices o entre colegas se acumulaba con base en la demostración cognitiva y explicativa de casos, mientras que en la sala clínica ello se hacía por medio del contacto directo con el paciente, con sus síntomas y tratamientos.

Sumado a la función de enseñanza, control interno y construcción de jerarquías de autoridad al interior del Instituto, los ateneos generaron notoriedad pública y reputación entre la comunidad de médicos local, pues el carácter encarnizado de las discusiones e intercambios desarrollados en los ateneos fue una de sus características más evocadas, lo que le dio su sello distintivo por lo cual eran famosos $(31,32)$.

En busca de una ecología común: las residencias y las unidades docentes hospitalarias. En los momentos iniciales del Instituto, a las modalidades informales de ingreso y cooptación de nuevos miembros, rápidamente se sumaron dos modelos institucionales de 
entrenamiento médico que funcionaron como mecanismos formales de reclutamiento y de formación médica: las residencias médicas y las unidades docentes hospitalarias (UDH). Estas fueron dos transformaciones en la enseñanza de la medicina que se plasmaron en nuevos planes de estudio de dicha carrera en la Facultad de Medicina. Primero, se implementaron las residencias médicas, entre 1958 y 1959, y luego, la UDH, en 1961. Ambos diseños respondieron a una concepción práctica del proceso de enseñanza-aprendizaje de la medicina, la residencia consistía en un entrenamiento de posgrado en el sitio hospitalario bajo una modalidad de tiempo completo, de 3 años de duración; la UDH implicaba la implementación de la cursada de los últimos tres años de grado (el ciclo clínico quirúrgico) en un hospital o grupo de hospitales, dentro del cual se debía hacer rotación, principalmente, por salas de clínica médica, cirugía, tocoginecología, pediatría y por las restantes especialidades $(33,34)$.

Las principales funciones del residente eran colaborar en la confección de la historia clínica, bajo la dirección del médico de cama, cooperar en trabajos de investigación clínica y en el trabajo experimental de laboratorio, asistir a los ateneos, realizar recorridas de sala, por lo menos tres diarias en su sector, hacer guardias y realizar autopsias. El horario de trabajo de los residentes era de jornada completa en la semana y los sábados solo hasta el mediodía (35).

La instauración del sistema de residentes significó un cambio sustantivo en múltiples planos. En lo atinente a la formación y entrenamiento médicos, tanto desde el punto de vista del aprendiz como del docente, introdujo nuevos desempeños, posiciones y destrezas para cada uno de ellos. La convivencia diaria entre residentes y jefes de residentes, médicos e instructores, con los pacientes, en el espacio de aprendizaje, conformado por el ambiente hospitalario, facilitaba y multiplicaba la posibilidad de incorporar los conocimientos teóricos y las habilidades y técnicas prácticas. A la vez les daba la posibilidad de estar en contacto con el material de estudio en tiempo y forma real (los pacientes), con la ventaja de asegurarse la socialización en el día a día, pudiendo consultar e intercambiar con sus docentes médicos y con sus compañeros, con quienes compartían jornadas completas de trabajo y aprendizaje.

Desde la óptica asistencial, la residencia sumó recursos humanos e introdujo nuevas figuras a la estructura de autoridad de la organización asistencial. En la base de la pirámide se hallaban los residentes menores, luego los mayores y finalmente el Jefe de residentes. A continuación seguían los médicos de cama y después los Jefes de sala de internación, conviviendo todos bajo el control último que quedaba en manos del Jefe de Clínica. En esta organización, si bien los residentes y Jefes de residentes ocupaban las posiciones más bajas en términos de recursos autoritativos y de decisión, eran quienes pasaban la mayor parte del tiempo con los enfermos y quienes finalmente tomaban, de hecho, muchas de las decisiones (más aún los Jefes de residentes).

Por su parte, la implementación de la unidad docente hospitalaria (UDH) implicó el inicio de un nuevo plan de estudios para los alumnos que habían terminado el ciclo básico, estipulando que los últimos tres años de la carrera de medicina (ciclo clínico y quirúrgico) se cursaran en el hospital. La carrera quedó dividida en dos ciclos: el básico y la UDH, de tres años cada uno. Las materias del ciclo básico continuaron siendo anuales y se cursaban en el edificio de la Facultad. Las materias de la UDH eran cursadas en hospitales (36). El criterio geográfico (espacial) del establecimiento de una ecología común para el cursado del ciclo clínico no solo ponía énfasis en la enseñanza y el entrena- 
miento situados en el mismo ámbito práctico del hospital sino también proyectaba a éstas en una modalidad de socialización intensiva y delimitada por una institución o por un grupo limitado de hospitales (evitando las rotaciones por múltiples espacios y su consecuente dispersión y dilapidación de recursos sociales $\mathrm{y}$ de conocimientos).

La UDH implicaba la asistencia diaria de cuatro horas al hospital y la realización de rotaciones por los distintos servicios correspondientes a las clínicas o a las especialidades. De esta manera, se cursaba una materia por vez y sus contenidos estaban compactados para ser enseñados en períodos de dos a doce semanas, según las asignaturas.

Según Lanari este nuevo plan no suponía sólo una distinta ordenación de materias sino que se proyectaba como una modificación sustancial del proceso de formación del médico (37). Para ello se habían introducido dos cambios: (i) el estudio de la clínica (fragmentado hasta entonces en semiología, patología interna y clínica médica) y el estudio de la cirugía (separado en técnica quirúrgica, patología externa y clínica quirúrgica) ahora confluían en un proceso continuo con los mismos docentes y durante un período de tres años; (ii) la formación en un mismo sitio hospitalario (evitando la movilización por distintos nosocomios según ocurría con las rotaciones del internado previamente).

\section{Conclusiones}

En este trabajo hemos reflexionado en torno a la espacialidad como algo más que una entidad física, viéndola como el resultado de un conjunto de prácticas en relación con el campo particular de la investigación clínica médica, desarrollada en el Instituto de Investigaciones Médicas. En parte, se intenta responder a las preguntas: ¿qué significó construir, aplicar y trasmitir conocimiento en un lugar particular como el Instituto
Lanari?, ¿qué tipo de actividades le dieron identidad hacia adentro y hacia afuera?

La interconexión espacial y práctica entre el laboratorio y la sala, entre la cultura experimental y la clínica, se reflejaron en el mapa edilicio general (las dos alas, el bar, el estacionamiento), en los espacios de formación y socialización (como los ateneos, las residencias, las unidades hospitalarias), en la diferenciación e interrelación entre especialidades y secciones (trasplantes, diálisis, hematología, inmunología), en los roles profesionales de investigador y clínico en una misma persona, en los pacientes (como pacientes y muestra) y en la construcción de autoridad y jerarquía puestas en juego en las actividades educativas, de investigación y asistencia llevadas adelante en la institución.

La Sección de trasplantes renales y el servicio de diálisis fueron espacios por los que el Instituto se constituyó como un lugar de referencia y reputación nacional y regional y a la vez aquellos funcionaron como constructores de identidad y autoridad científica hacia adentro del Instituto, en el doble proceso de legitimación que tiene todo lugar del saber, intramuros y de cara a la sociedad y otras instituciones científicas (1). Asimismo, el desarrollo de estos espacios cognitivos de investigación y tratamiento (las relaciones de colaboración y formación de recursos humanos con el grupo de Boston, el traslado de técnicas de allí y su puesta en funcionamiento en el Instituto) mostraron otra tensión espacial en juego: el enraizamiento local de los conocimientos científicos conviviendo con dependencias y relaciones internacionales (1-3).

Los ateneos semanales constituyeron un espacio de socialización de médicos y residentes a partir de razonamientos, diagnósticos y tratamientos y a la vez fueron el lugar desde donde construir una clara jerarquía de autoridad y responsabilidad en cuanto a la toma final de 
decisiones en cada caso tratado: la del director del Instituto y, luego, la de los respectivos jefes de sección. Este escalonamiento de autoridad y responsabilidad era trasladada a otras relaciones entre jefes y subalternos en ámbitos diferentes al del espacio de los ateneos. Por ejemplo, en la sala de internación, en los laboratorios y en los espacios de docencia, con la implementación del sistema de residencias y de la unidad docente hospitalaria (UDH). La construcción de autoridad cognitiva y social y la conformación de relaciones de jerarquía implicaron de este modo una forma de espacialidad (o, en otras palabras, se configuraron en determinados espacios de interacción).

Con las residencias y la UDH se conformó un mecanismo de reclutamiento hospitalario integrado y continuado (desde el último ciclo del grado hasta el de posgrado), que se perfilaba como paso previo o plataforma desde la cual poder diseñar, extender y consolidar una carrera médica hospitalaria asentada en la imbricación de destrezas de atención, docencia e investigación dentro del ámbito hospitalario. Ambas, las residencias y la UDH fueron transformaciones pedagógicas y espaciales en torno a la formación y al entrenamiento médico, dentro de una ideología institucional que privilegiaba la imbricación entre las tareas de enseñanza, atención e investigación. Es decir, entre la transmisión, el uso y la producción de conocimientos, entendido y puesto en práctica como un proceso espacial continuo y a la vez discreto.

\section{Referencias}

1. Henke, C Gieryn, T. Sites of scientific practices: the enduring importance of place. En: Hackett E, Amsterdamska O, Lynch M, Wajcman J (editores). The Handbook of science and technology studies, 3 . $^{\mathrm{a}} \mathrm{ed}$. Cambridge and London: The Mit Press; 2008, p. 353-86.

2. Amsterdamska O. Practices, People and Places. En: Hackett E, Amsterdamska O, Lynch, M Wajcman J (editores). The Handbook of science and technology studies, 3. ${ }^{a}$ ed. Cambridge and London: The Mit Press; 2008, p. 205-9.

3. Salvatore R. Los lugares del saber. Contextos locales y redes trasnacionales en la formación del conocimiento moderno. Rosario: Beatriz Viterbo editora; 2007.

4. Hollister H. The planning and construction of High School Buildings. University of Illinois Bulletin 1916;14(8):12-41.

5. Peters P. Escuelas Superiores. Centros de investigación. Barcelona: Editorial Gustavo Gili; 1972.

6. Smith H. Buildings for Research, Architectural Record Book. s.d.: Dodge Corporation; 1958.

7. Gentile E. Ciudad Universitaria. En: Liernur JF, Aliata F (editores.). Diccionario de Arquitectura en la Argentina, Buenos Aires: AGEA; 2004. p. 1-45.

8. Shinn T. Jerarquías de investigadores y formas de investigación. REDES 2007;13(25):119-63.

9. Corsín-Jiménez A. On Space as a Capacity. Journal Royal Antropological Institute. (N.S.) 2003;9:137-53.

10. Giddens A. La constitución de la sociedad. Bases para la teoría de la estructuración, Buenos Aires: Amorrortu; 2011.

11. Romero L. Conformación y desarrollo de una tradición de investigación clínica médica: Alfredo Lanari y el Instituto de Investigaciones Médicas de la Universidad de Buenos Aires [tesis de doctoral], [Buenos Aires]. FLACSO; 2011.

12. Buschini J. Renovación institucional y modernización científica: la creación del Instituto de Investigaciones Hematológicas a mediados de la década de 1950. Salud Colectiva 2013;9(3)317-34. 
13. Romero L, Buschini J. La construcción de un departamento científico en un proceso intensivo de modernización académica: el caso de la física en Ciencias Exactas (UBA), 1955-1966; en La construcción de la ciencia académica: actores, instituciones y procesos en la Universidad argentina del siglo xx; Buenos Aires: Ed. Biblos; 2010, p. 165-86.

14. Gaudilliére P. Inventer la biomédecine. La france, $\mathrm{l}^{\prime}$ amérique et la production de savoirs du vivant. Paris: éditions la découverte; 2002.

15. De Asúa M. La formación del médico en la República Argentina. Análisis histórico-sistemático del perfil curricular de las Facultades de Medicina de la Argentina desde su creación hasta 1982 [tesis de doctoral]. [Buenos Aires]: Universidad de Buenos Aires; 1984.

16. Facultad de Medicina, Universidad de Buenos Aires (UBA). Actas de Consejo Directivo. Resolución 564 y 639/57 (1957).

17. Martín R, Barcat JA Molinas F (editores). Alfredo Lanari. Vocación y convicción. Reflexiones sobre la investigación, el futuro de la medicina y otros escritos. Buenos Aires: Fundación Alfredo Lanari; 1995.

18. Investigador del IIM, Sección Nefrología, entrevista personal, 7 de julio de 2010.

19. Agrest A. El Instituto de Investigaciones Médicas. En: Rotunno C, Díaz de Guijarro E. (editores). La construcción de lo posible. Buenos Aires: El Zorzal; 2003. p. 203-8.

20. Médico del IIM, Sección Radioisótopos, entrevista personal, 15 de diciembre de 2008.

21. Ciencia Nueva. Investigación en clínica médica. Reportaje a Alfredo Lanari. Ciencia Nueva 1970;(3)26-33.

22. Investigador del IIM, Sección Metabolismo, entrevista personal, 25 de febrero de 2009.

23. Ruiz-Guiñazú A. El riñón artificial. Funcionamiento y aplicación clínica. La Prensa Médica Argentina 1957;XLIV(52)3746-53.

24. Romero L. Origen y desarrollo de la diálisis y los trasplantes renales en la Argentina: dinámicas científico técnicas, institucionales y sociales. Asclepio 2012;64(2)565-92.

25. Löwy I. The impact of medical practice on biomedical research: the case of human leucocyte antigens studies. Minerva 1987a;25(1-2)171-200.

26. Löwy I. Choix scientifiques et Choix éthiques dans le traitement de la maladie rénale terminale, Information sur les Sciences Sociales (SAGE, Londres); 1987b, p. 577-605.

27. Rodo J. Trasplantes renales en la Argentina. Su historia. Trasplantes 1997;3(2):2-5.

28. Arce M. Historia de la Hemodiálisis y Trasplante renal en la República Argentina. Revista de Nefrología, Diálisis y Trasplante 1980;0(1):1-3.

29. Manzor D. Historia de la Nefrología. Entrevistas realizadas a los anatomopatólogos Dr. Osvaldo I. Falcón y Dr. Roberto Iotti por el Dr. Daniel N. Manzor. Revista de nefrología, diálisis y trasplante 2006;26(1):5-9.

30. Investigador del IIM, Sección Anatomía Patológica, entrevista personal, 16 de diciembre de 2008.

31. Investigador del IIM, Sección Hematología. Entrevista personal, 2 de marzo de 2009.

32. Investigador del IIM, Sección Inmunología. Entrevista personal, 20 de diciembre de 2007.

33. Ceitlin J. Problemas de Educación Médica. Organización docente actual en la escuela de medicina. Plan de estudios. Medicina Panamericana 1962;xviII(2):35-9.

34. Quirno N. Presente y Futuro de los médicos residentes en la Argentina; su ubicación en los servicios: obligaciones docentes y ventajas asistenciales. Revista de la AMA 1963;77(8):403-5.

35. Lanari A. Residencias hospitalarias en Clínica Médica. Revista de la AMA 1960;74(3):98-101.

36. Munist L. Memoria del Decano Luis Munist. Buenos Aires: Facultad de Medicina, Universidad de Buenos Aires; 1963.

37. Lanari A. Consideraciones sobre la unidad hospitalaria y sus resultados a los 6 años de su creación. El Día Médico 1967;39(59):1166-9. 